\title{
FEATURE FUSION BASED ON AUDITORY AND SPEECH SYSTEMS FOR AN IMPROVED VOICE BIOMETRICS SYSTEM USING ARTIFICIAL NEURAL NETWORK
}

\author{
Youssouf Ismail Cherifi ${ }^{1, *}$ and Abdelhakim Dahimene ${ }^{1,2, \dagger}$ \\ ${ }^{1}$ University M'hamed Bougara, Boumerdes, Algeria \\ ${ }^{2}$ Signal and System laboratory, Boumerdes, Algeria
}

\begin{abstract}
In today's world, identifying a speaker has become an essential task. Especially for systems that rely on voice commands or speech in general to operate. These systems use speaker-specific features to identify the individual, features such as Mel Frequency Cepstral Coefficients, Linear Predictive Coding, or Perceptual Linear Predictive. Although these features provide different representations of speech, they can all be considered as either auditory system based (type 1) or speech system based (type 2).

In this work, a method for improving existing voice biometrics system is presented. A method fusing a type 1 feature with a type 2 feature is implemented using artificial neural network and evaluated on in-campus recorded data set. The obtained results demonstrate the potential of our approach in improving voice biometrics system, regardless of the underlying task being speaker identification or verification.
\end{abstract}

\section{KEYWORDS}

Speech Processing, Neural Network, Pattern Recognition, Speaker Recognition, Feature Extraction

\section{INTRODUCTION}

It is a well-known fact that speech itself contains several levels of information conveyed to the auditor. Mainly, speech is used to communicate a message to the auditor, a message that is carried in words. However, speech also contains information about the speaker himself, this is due to the way it is produced. This information could be for instance the speaker's gender, emotions, age, origin and obviously identity. Recognizing a person's identity by analyzing a portion of his speech is known as Speaker Recognition (Reynolds, 2002).

Depending on the number of identities involved, speaker recognition can be one of two tasks: speaker verification or speaker identification. In speaker verification, the objective is to verify that the speaker is the exact person he claims to be. On the other hand, speaker identification goal is to assert the identity of a speaker among a closed set of known speakers. In addition to the number of speakers involved, speaker recognition tasks can be either text-dependent or text-independent based on the user level of control and cooperation.

The development of speaker recognition methods and techniques has been an active field of research for well over five decades and continues to be. These methods have extended from using spectrogram comparisons to basic template matching, to dynamic time-warping algorithms, to more advance statistical pattern recognition algorithms such as Artificial Neural Networks (ANNs) and Hidden Markov Models (HMMs). In fact, most of the research that has been done in order to improve the accuracy of speaker recognition tasks focuses on the development of high-performance pattern recognition algorithms or the adjustment of existing algorithms. An example of this would be the use of Gaussian Mixture Model (GMM) (Chakroum et al, 2016) or Artificial Neural Network (ANN) (Srinivas et al, 2014) to obtain a better recognition rate.

\footnotetext{
youcef.ismail.cherifi@univ-boumerdes.dz

a.dahimene@univ-boumerdes.dz
} 
Continuous research and effort are ongoing, involving the combination of two modelling techniques (Al-Shayea \& Al-Ani, 2016. Chakroborty \& Saha, 2009. Singh et al, 2016. Awais et al, 2014) or the implementation of specific hardware (Gaafar et al, 2014).

Having a better recognition rate does not depend only on the modelling techniques, as shown in previous work (Paliwal et al. in 2010), where a better is obtained by adjusting the width of the window at which the feature was extracted. Similar conclusions were deducted (Gulzar et al. 2014; Dave, 2013), but instead of adjusting the frame width, the effect of changing the set of features entirely was studied. In some other work (Eringis and Tamulevicius, 2014) it was shown that by adjusting the frame width and increment for different features, an improvement of $4.15 \%$ (from $88.75 \%$ to $92.9 \%$ ) can be achieved.

Based on the aforementioned work, an improved speaker recognition scheme is suggested in this paper. This scheme has a high accuracy that makes it well suited for applications in various fields such as security and crime investigations.

\section{MATERIALS AND METHODS.}

\subsection{Proposed Approach}

The adopted approach for improving the recognition rate is to fuse two different types of features. The fused features are a combination of auditory system-based feature (type 1) with a speech system-based feature (type 2). To ensure that both features are kept isolated and not grouped under the same vector space, Artificial Neural Networks (ANN) algorithm is used to model the voice print. Using ANN, each set of features is connected to a dedicated input layer and are only fused/merged at the hidden layer (Figure 1).

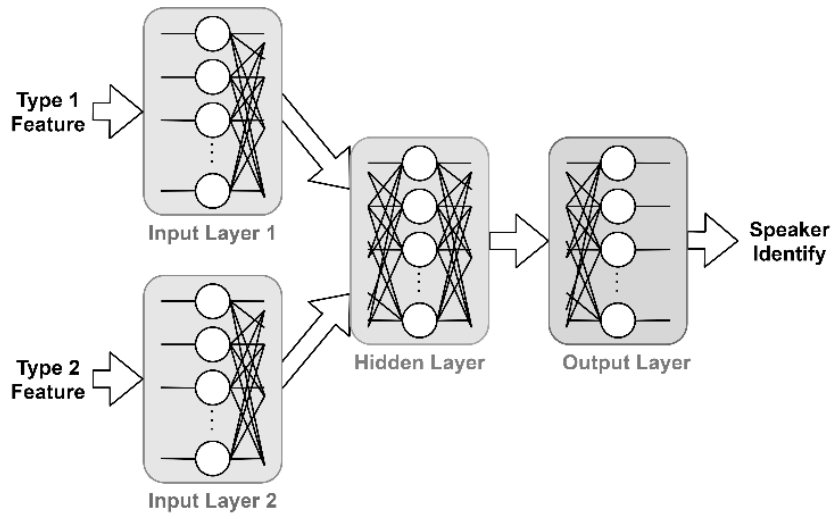

Figure 1 . The proposed system for feature fusion. Type 1 feature is auditory system-based feature and type 2 is speech system-based feature

The structure shown above was implemented using MATLAB 2017a Deep Learning Toolbox (Figure 2). Each of the input layer as well as the hidden layer contained 44 perceptrons, to account for all possible phonemes in the English language. While the output layer contained a number of perceptrons equivalent to the number of speakers.

To reduce the influence of extreme values or outliers in the dataset without having to remove them, a SoftMax function was used as the activation function for the output layer. For input layers and hidden layers perceptrons, the tangential sigmoid activation function was used. This function has a steeper derivative which make it a good candidate for extracting intermediary features (Meena et al, 2011).

To train the structures shown above, the conjugate gradient backpropagation algorithm is used to reduce the Sum of Square Errors (SSE) between the outputs of the network and a vector of desired targets. This algorithm has a better accuracy when compared with other algorithms as shown in Table 1. 


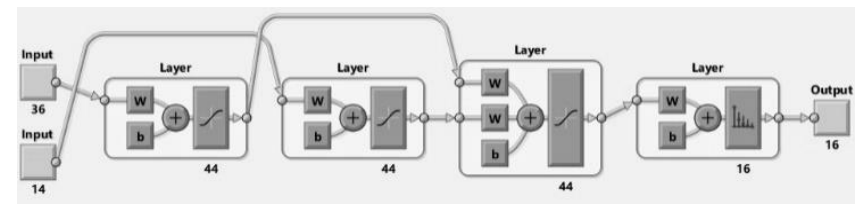

Figure 2. Feature fusion ANN structure

Table 1. Performance of different training functions in MATLAB's NN toolbox (Vacic, 2015)

\begin{tabular}{lcccccccc}
\hline \multirow{2}{*}{ Function name } & \multicolumn{2}{c}{ training } & \multicolumn{2}{c}{ validation } & \multicolumn{2}{c}{ testing } & \multicolumn{2}{c}{ time } \\
\cline { 2 - 9 } & mean & stdev & mean & stdev & mean & stdev & mean & stdev \\
\hline trainb & 0.6456 & 0.7246 & 0.6302 & 0.6946 & 0.6386 & 0.7081 & 2.511 & 3.3835 \\
trainbfg & 0.0096 & 0.0032 & 0.0199 & 0.0084 & $\mathbf{0 . 0 2 0 9}$ & 0.0046 & 7.3219 & 4.5702 \\
trainbr & 7.6088 & 3.5328 & 18.9761 & 10.219 & 149.8294 & 32.2893 & 18.5063 & 8.927 \\
traincgb & 0.0102 & 0.0026 & 0.0193 & 0.0069 & $\mathbf{0 . 0 2 0 3}$ & 0.0059 & 4.3389 & 1.886 \\
traincgf & 0.0112 & 0.0033 & 0.0199 & 0.0091 & $\mathbf{0 . 0 2 0 2}$ & 0.0051 & 4.9752 & 2.4127 \\
traincgp & 0.0114 & 0.003 & 0.0213 & 0.0093 & $\mathbf{0 . 0 2 1 6}$ & 0.0045 & 4.0544 & 1.9337 \\
traingd & 0.0265 & 0.0055 & 0.0332 & 0.0099 & 0.0323 & 0.0029 & 13.003 & 4.4432 \\
traingdm & 0.5528 & 0.34 & 0.5556 & 0.3221 & 0.5592 & 0.3499 & 1.2875 & 0.3697 \\
traingda & 0.0244 & 0.0063 & 0.0293 & 0.0084 & 0.0310 & 0.0037 & 5.2 & 2.222 \\
traingdx & 0.0394 & 0.0312 & 0.0448 & 0.0317 & 0.0445 & 0.0274 & 5.4219 & 3.526 \\
trainlm & 0.0065 & 0.0027 & 0.0199 & 0.0066 & $\mathbf{0 . 0 2 3 1}$ & 0.0037 & 8.5762 & 3.494 \\
trainoss & 0.013 & 0.0038 & 0.0204 & 0.0081 & $\mathbf{0 . 0 2 0 5}$ & 0.0035 & 5.1703 & 2.8221 \\
trainrp & 0.0137 & 0.0045 & 0.0207 & 0.0059 & 0.0229 & 0.0035 & 7.4954 & 3.8277 \\
trainscg & 0.0114 & 0.0035 & 0.0213 & 0.0109 & $\mathbf{0 . 0 2 1 8}$ & 0.0073 & 4.3171 & 1.7394 \\
\hline
\end{tabular}

Overall, 3 set of features were extracted to test the effectiveness of the suggested approach. The extraction was also done in MATLAB using the Auditory Toolbox (Malcom Slaney) and VOICEBOX (Mike Brooks).

\subsubsection{Mel Frequency Cepstral Coefficient}

The MFCC are among the most dominant and common features in the field of Natural Language Processing. They represent the short-term power spectrum of a given audio clip but what set them apart from the cepstrum is the fact that use a Mel-scaled filter banks. These filter banks are equally spaced, and they approximate the human auditory system more than the linearly spaced bands used in the cepstrum. This makes MFCC the best candidate as a type 1 feature.

To extract MFCC from an audio signal, the signal is processed as shown in Figure 3. At the end of this pipeline 13 coefficients were extracted. The $0^{\text {th }}$ coefficient is discarded since it does not contain any significant information. The delta and delta-delta were added to the remaining 12 coefficients to ensure that the model is getting sufficient information about the fluctuation of the signal.

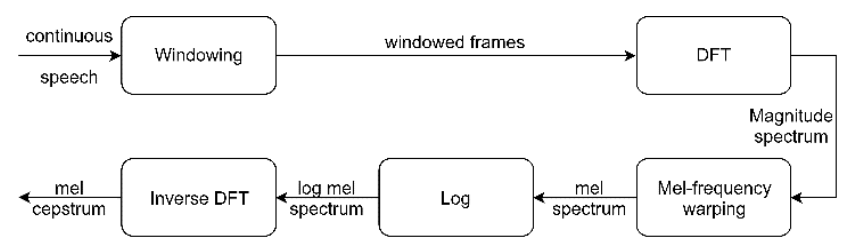

Figure 3. MFCC derivation

$$
\begin{gathered}
\Delta_{\mathrm{k}}=\mathrm{f}_{\mathrm{k}}-\mathrm{f}_{\mathrm{k}-1} \\
\Delta \Delta_{\mathrm{k}}=\Delta_{\mathrm{k}}-\Delta_{\mathrm{k}-1}
\end{gathered}
$$




\subsubsection{Linear Predictive Coding}

LPC is a good candidate for speech analysis due to the nature of the speech generation process. The entire process can be represented by a digital filter as modelled in Fig 4 which makes LPC one of the most powerful type 2 features for speech analysis (Buza et al, 2006).

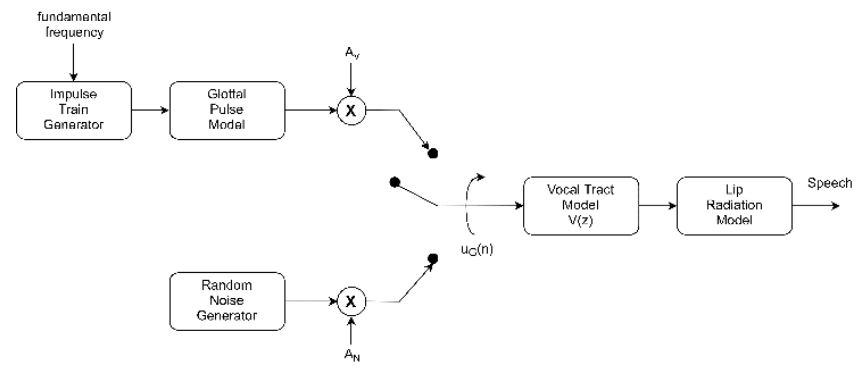

Figure 4. Speech Production Model. $A_{v}$ is the voiced sound gain, $A_{n}$ is the unvoiced sound gain and $u_{G}(n)$ is voiced/unvoiced switching function

To extract LPC from an audio signal, the signal is first framed using short time Hamming windows. For each frame 14 formants or coefficients are extracted. This ensures that all possible speech segments (voiced and unvoiced) are covered and for both genders as well. Formants are extracted by computing coefficients that link the current speech sample with the previous samples with the same window. This can be expressed by the following equation:

$$
s(n)=\sum_{k=1}^{p} \alpha_{k} s(n-k)
$$

Where $\mathrm{s}$ is the speech signal, $\mathrm{n}$ is the sample point, $\alpha$ is the formant and $\mathrm{p}$ is the number of the required formants (14 in our case).

\subsubsection{Perceptual Linear Prediction}

Similarly to LPC, PLP models the human speech based on how speech is produced. PLP differs from LPC on the fact that it discards irrelevant information of the speech and thus approximating the human speech system in a way that improves the recognition rate.

\subsection{Data Collection}

To carry out this work, sixteen participants were recruited from the Institute of Electrical and Electronic Engineering (IGEE) to record a corpus of 51 to 54 English sentences each. All participants were PhD students with ages ranging from 24 to 27 ( 8 females, 8 males; mean age: $25.37 \pm 0.88$ ). They were all non-native English speakers from Algeria, with a relatively high level of proficiency in English (mean TOEFL score: $89.75 \pm 6.75$ with a mean of $26.06 \pm 2.93$ in the speaking section).

All recordings were carried out in the same room located within the institute, $(6.0 \mathrm{~m}(\mathrm{~L}) \mathrm{x} 3.5 \mathrm{~m}$ (W) $x 4 \mathrm{~m}(\mathrm{H})$ as shown in Figure 5). Participants were sitting on a chair, facing a wall at a distance of $0.75 \mathrm{~m}$ with a monitor in front of them displaying the sentence to be read. The recording device (Honeywell CN51 Personal Digital Assistant (PDA)) was placed between the monitor and the participant.

The recorded sentences differ from one student to the other to ensure that the task remains text-independent and the collaboration of the speaker to a minimum. 


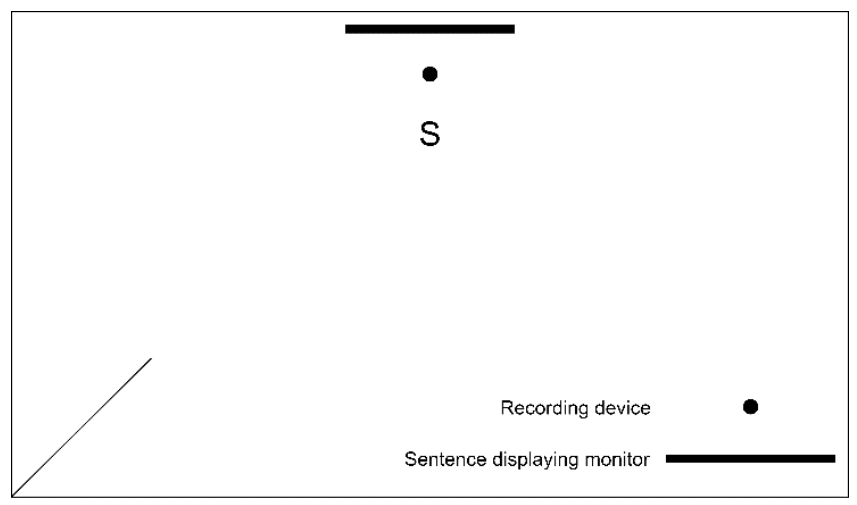

Figure 5. Recording room layout. $\mathrm{S}$ is the speaker/participant

To demonstrate that the approach is effective for a wider range of scenario, the LibriSpeech ASR corpus (Vassil et al, 2015) was implied. The corpus however was only used to confirm the recognition accuracy and was not used for all procedures due to hardware limitations.

\subsection{Procedures}

To assess the performance of the feature fusion approach, a specific pipeline was put in place.

\subsubsection{Tuning the Parameters for Feature Extraction}

The first step for improving the recognition rate is to adjust the frame width and increment during feature extraction. This improvement has already been shown (Paliwal et al, 2010. Eringis \& Tamulevicius, 2014) given that these parameters determine whether the modeling algorithms are getting a sufficient level of information from the speech segment as input.

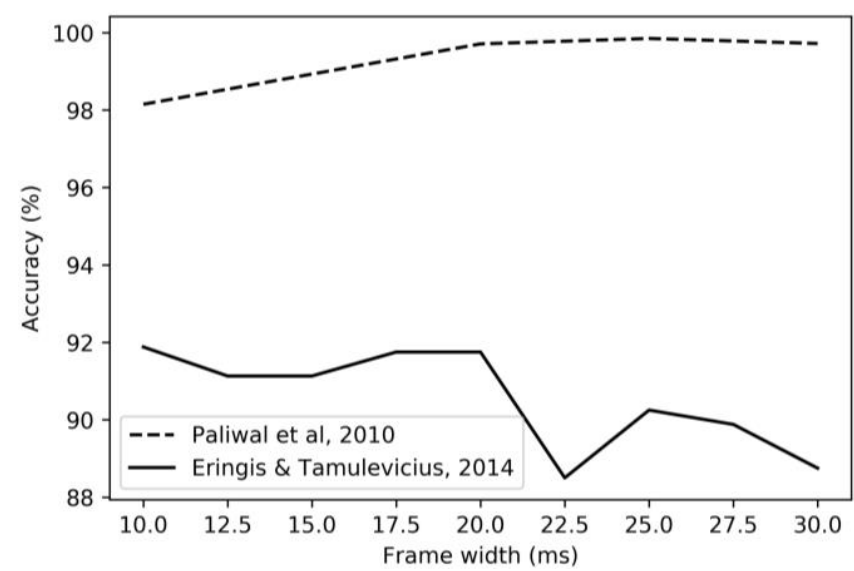

Figure 6. The effect of adjusting the frame width during MFCC extraction on the overall accuracy, blue for the work done by Paliwal et al, 2010 and orange for the work done by Eringis and Tamulevicius, 2014

There is some discrepancy in the literature regarding the frame width values which optimize accuracy (see Figure 6). To confirm which frame width to choose, the structure shown in Figure 7 is trained with MFCC features extracted using different frame widths. Thirty random segments for each speaker in the data set are used for training while the remaining 21 segments are used for testing. This training was carried out in a cross-validation setup to avoid any overfitting due to the complex nature of chosen model. Once the overall accuracy was obtained, the frame width was increased by $5 \mathrm{~ms}$. This procedure was repeated until a frame width of $30.0 \mathrm{~ms}$ was reached.

The second parameters to consider for tuning the feature extraction was the frame increment. For this, 3 values were considered: Overlapping (50\%), Slightly-Overlapping (75\%) and Non-Overlapping (100\%). For each of these values the same network shown in Fig 7 was trained with 30 cross-validated speech segments. 


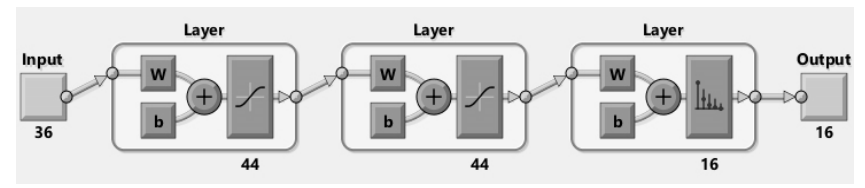

Figure 7. The single feature ANN structure

\subsubsection{Fusing, Training and Testing}

To study the effect of fusing features on the overall recognition rate, the structure shown in Figure 2 is trained using two features extracted at the optimal frame width and increment. For each speaker, 30 random segments are used for training while the remaining 21 segments are used for testing. The selected segments were cross-validated and the resulting performance was compared to that of training the structure shown in Figure 7 using a single feature extracted at the same optimal parameters.

The same procedure was repeated for the LibriSpeech corpus. To ensure that the approach works on a larger dataset (921) and on native English speakers. Although the corpus contains more than 51 segments for each speaker, only 51 segments were used between training and testing similarly to the recorded corpus.

\section{RESULTS \& DISCUSSION}

When adjusting for the frame width during feature extraction, the best recognition rate was obtained for a frame width of $10 \mathrm{~ms}$, which coincides with the results obtained by Eringis and Tamulavicius, 2014. These results are depicted in Figure 8. This pattern of improvement was found across all three features that were extracted.

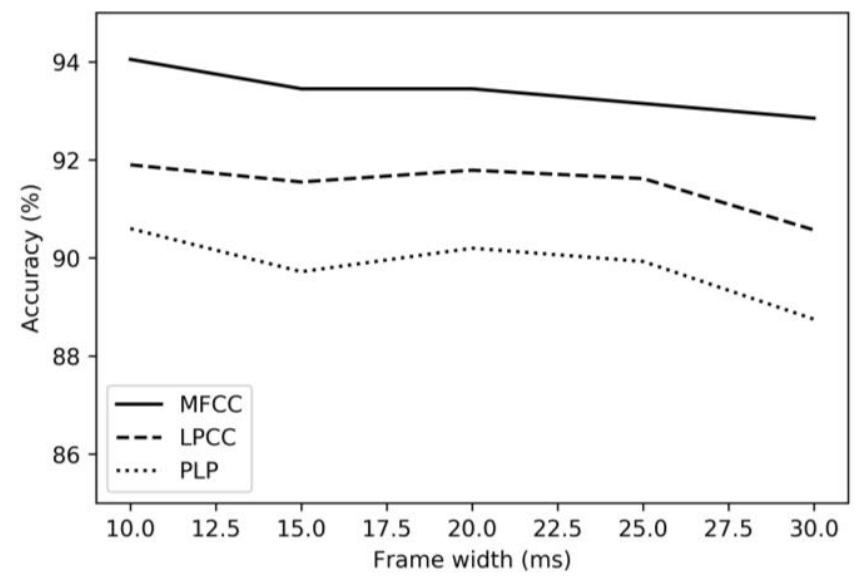

Figure 8 . The results of frame width tuning tasks

Examining the 3 different frame increments for the optimal frame width of $10 \mathrm{~ms}$, we found that a frame increment of $75 \%$ provided the highest recognition rate on average compared to the $50 \%$ and $100 \%$ increments (see Fig 9). This implies that by only adjusting the extraction parameters, 3 additional speech segments were correctly identified. 


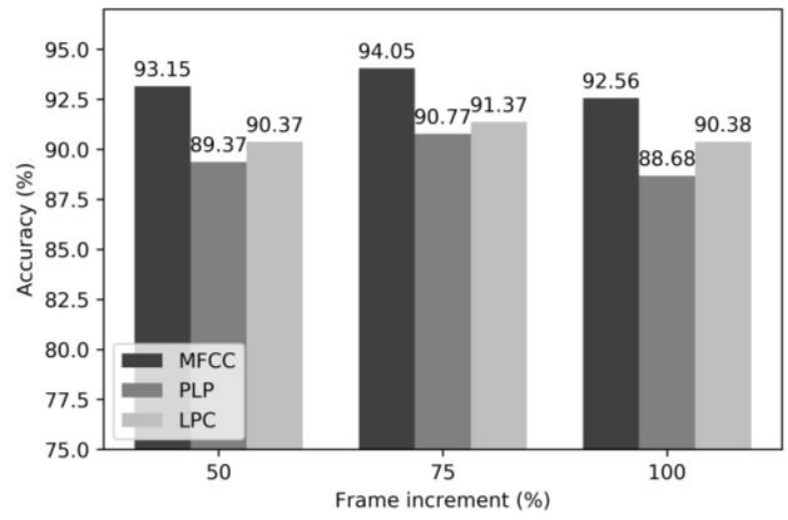

Figure 9. The results of frame increment tuning task

The fusion of type 1 and type 2 features improved the accuracy of the recognition tasks. As seen in Figure 10, any combination of type 1 and type 2 feature would result in a better recognition rate than using just a single feature. The best result was obtained when combining MFCC and LPC (99.4\% accuracy), this is due to the fact that these two sets of features are uncorrelated.

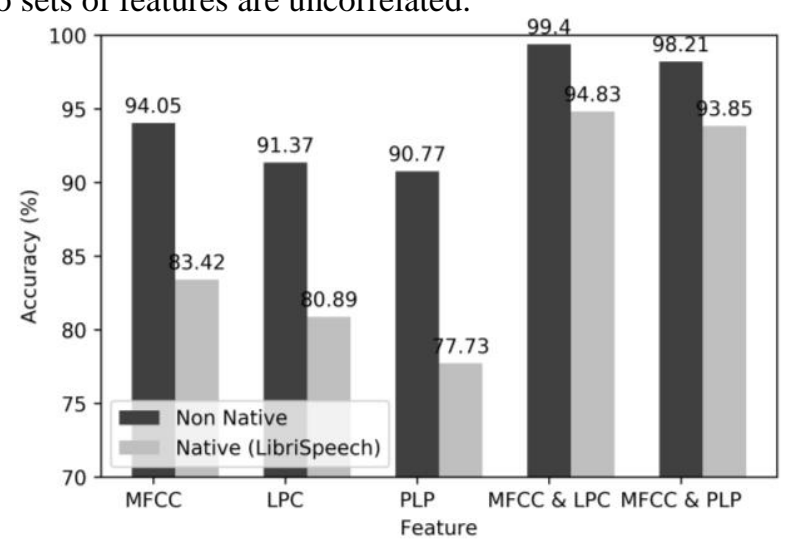

Figure 10. Feature fusion effect on the overall accuracy for speaker recognition. MFCC: Mel-frequency cepstral coefficients; LPC: Linear predictive coding; PLP: Perceptual linear predictive

Figure 10 also demonstrates that the approach works well for native speakers and for a larger set of speakers. In fact, the improvement obtained when testing for the larger corpus was higher. This was due to the fact that this approach created higher dimensionality allowing for a better discrimination. Of course, the approach resulted in a slight increase in the training time. Fig 11 shows the required training time for each of the trials described in section 2.5. These results were obtained using the 4710MG i7 CPU with a RAM of $8 \mathrm{~GB}$.

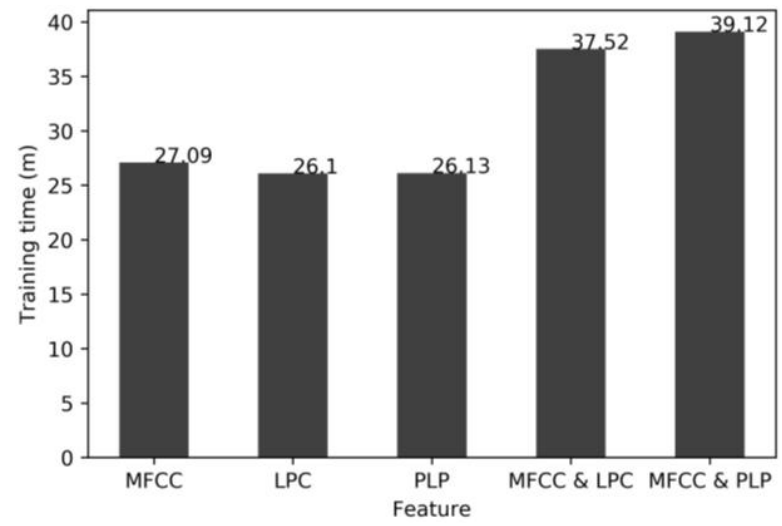

Figure 11. Feature fusion effect on the required training time for speaker recognition. MFCC: Mel-frequency cepstral coefficients; LPC: Linear predictive coding; PLP: Perceptual linear predictive 
The difference in training time between the best performing single feature structure and the best performing fused features structure is 10 minutes and 25 seconds. This means an increase of $38.50 \%$ in training time for an improvement of 5.35\% in speaker-recognition accuracy if we consider only the effect of feature fusion without the parameter tuning and $6.55 \%$ when considering the parameters tuning. This is in fact very significant if we consider and application such as criminal investigation where $6.55 \%$ means that 22 cases or suspects are correctly being recognized using, for example, a phone call.

In addition, the training time can be significantly reduced, if a better hardware is used such as a more performing GPU or/and by reducing the duration of the recordings that are used for training the model as feature fusing allows to recognize the speaker much earlier as shown in Figure 12 and Table 2.

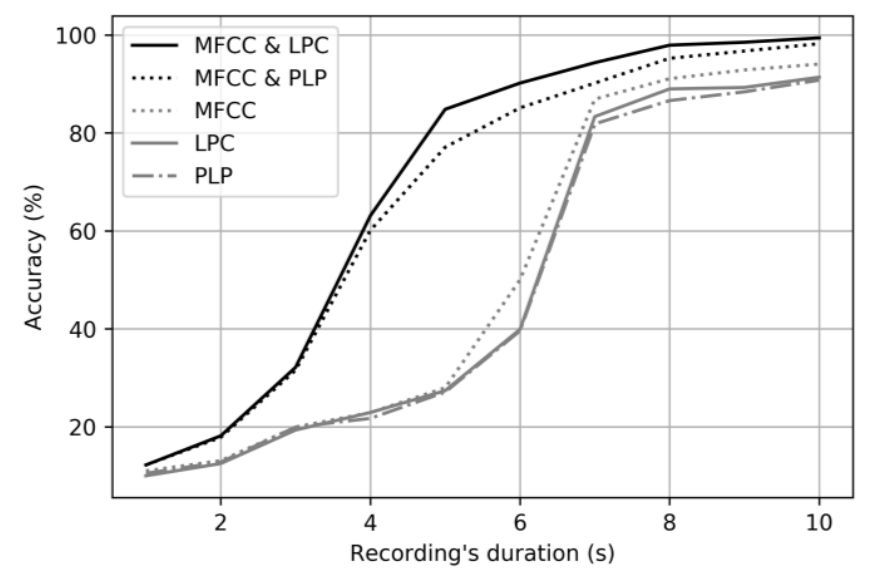

Figure 12. Speaker recognition accuracy over time. MFCC: Mel-frequency cepstral coefficients; LPC: Linear predictive coding; PLP: Perceptual linear predictive

Table 2. Speaker recognition accuracy over time. MFCC: Mel-frequency cepstral coefficients; LPC: Linear predictive coding; PLP: Perceptual linear predictive

\begin{tabular}{lcccccccccc}
\hline \multirow{2}{*}{ Input } & \multicolumn{10}{c}{ Time (s) } \\
\cline { 2 - 13 } & $\mathbf{1}$ & $\mathbf{2}$ & $\mathbf{3}$ & $\mathbf{4}$ & $\mathbf{5}$ & $\mathbf{6}$ & $\mathbf{7}$ & $\mathbf{8}$ & $\mathbf{9}$ & $\mathbf{1 0}$ \\
\hline MFCC & 11.01 & 13.09 & 19.94 & 22.91 & 27.97 & 50 & 86.9 & 91.07 & 92.85 & 94.05 \\
\hline LPC & 10 & 12.5 & 19.34 & 22.91 & 27.38 & 39.88 & 83.33 & 88.89 & 89.28 & 91.37 \\
\hline PLP & 10.5 & 12.79 & 19.94 & 21.72 & 27.08 & 39.58 & 81.84 & 86.60 & 88.39 & 90.77 \\
\hline MFCC \& LPC & 12.20 & 18.15 & 32.14 & 63.09 & 84.82 & $\mathbf{9 0 . 1 7}$ & $\mathbf{9 4 . 3 4}$ & 97.91 & 98.57 & 99.4 \\
\hline MFCC \& PLP & 12.20 & 17.85 & 31.54 & 60.11 & 77.08 & 85.11 & 90.17 & 95.23 & 96.72 & 98.21 \\
\hline
\end{tabular}

By using 6 seconds of recording the model was able to reach 90.17\% when fusing MFCC with LPC. What is even more important about these results is the fact that the fusion approach was able to outperform the single feature approach by utilizing 7 seconds out of the provided 10 seconds of recording, this reduced training time in the fusion approach from 37 minutes and 32 seconds to 24 min and 41 seconds. This time is less than the time required to train the model for any of the features independently. These results are significant when taking into consideration the applications of speaker recognition. For fields such as security, this means less data storage, and for crime investigation this means the ability to identify a suspect even if the provided audio is short.

\section{CONCLUSION}

The speech signal does not only convey a message, it conveys information about the speaker themselves, their gender, origins, health and age. The aim of this work was to improve the task of recognizing a person based on speech segments. 
The approach we used proved to be very effective in improving the speaker recognition rate. This improvement was achieved with the use of two sets of features instead of just one. These two feature sets are completely uncorrelated and each one represents different characteristics of the speech signal. The drawback of such approach is the long training time (over 39 minutes for 480 speech segments). Nevertheless, this can be mitigated by using an approach such as deep features, where the trained model input and hidden layers are kept. The output layer however could be replaced by a less time demanding classification or clustering technique such as Support Vector Machines (SVM) or Gaussian Mixture Models (GMM).

The demonstrated approach improved the recognition rate by $6.55 \%$, which means it recognized 22 more segments out of the 336 speech segments used for testing. This was achieved at the cost of a 10 minutes and 26 seconds increment in the training time and no change in the testing time.

\section{REFERENCES}

Al-Shayea Q.K., and Al-Ani M.S., 2016. Speaker Identification: A Novel Fusion Samples Approach. In International Journal of Computer Science and Information Security, Vol. 14, No. 7, pp 423-427.

Awais M., Mansour A., and Ghulam M., 2014. Automatic Speaker Recognition Using Multi-Directional Local Features (MDLF). In Arabian Journal for Science and Engineering, Vol. 39, No. 5, pp 3379-3811.

Buza O., Toderan G., Nica A., and Caruntu A., 2006. Voice Signal Processing for Speech Synthesis, In International Conference on Automation, Quality and Testing, Robotics. Cluj-Napora, Romania, pp. 360-364.

Chakroborty S. and Saha G., 2009. Improved Text-Independent Speaker Identification using Fused MFCC \& IMFCC Feature Sets based on Gaussian Filter. In International Journal of Electrical, Computer, Energetic, Electronic and Communication Engineering, Vol. 3, No. 11, pp 1974-1982.

Chakroum R., Zouari L.B., Frikha M., and Ben Hamida A., 2016. Improving Text-independent Speaker Recognition with GMM. In International Conference on Advanced Technologies for Signal and Image Processing, Monastir, Tunisia, pp. 693-696.

Dave N., 2013. Feature Extraction Methods LPC, PLP and MFCC in Speech Recognition. In International Journal for Advance Research in Engineering and Technology, Vol. 1, No. 6 , pp 1-5.

Eringis D., and Tamulevicius G., 2014. Improving Speech Recognition Rate through Analysis Parameters. In Electrical Control and Communication Engineering, Vol. 5, pp 61-66.

Gaafar T.S., Abo Baker H.M., and Abdalla M.I., 2014. An improved method for speech/speaker recognition. In International Conference on Informatics, Electronics \& Vision, Dhaka, Bangladesh, pp. 1-5.

Gulzar T., Singh A., and Sharma S., 2014. Comparative Analysis of LPCC, MFCC and BFCC for the Recognition of Hindi Words using Artificial Neural Networks. In International Journal of Computer Applications, Vol. 101, No. 12, pp 22-27.

Meena K., Subramaniam K., and Gomathy M., 2011. Gender Classification in Speech recognition using Fuzzy Logic and Neural Network. In The International Arab Journal of Information Technology, Vol. 10, No. 5, pp 477-485.

Paliwal K.K., Lyons J.G., and Wojcicke K.K., 2011. Preference for 20-40 ms window duration in speech analysis. In International Conference on Signal Processing and Communication Systems, Gold Coast, Australia, pp. 1-4.

Reynolds D.A., 2002. An Overview of Automatic Speaker Recognition Technology. In International Conference on Acoustics, Speech, and Signal Processing, Orlando, Florida, USA, pp. 4072-4075.

Singh S., Assaf M.H., Das S.R., Biswas S.N., Petriu E.M., and Groza V., 2016. Short Duration Voice Data Speaker Recognition System Using Novel Fuzzy Vector Quantization Algorithm. In International Instrumentation and Measurement Technology Conference Proceedings, Taipei, Taiwan, pp. 1-6.

Srinivas V., Santhi C.R. and Madhu T., 2014. Neural Network based Classification for Speaker Identification. In International Journal of Signal Processing, Image Processing and Pattern Recognition, Vol. 7, No. 1, pp 109-120.

Vacic V., 2015. Summary of the training functions in Matlab's NN toolbox. In MSc, University of California, Riverside, California, USA.

Vassil P., Guoguo C., Daniel P., and Sanjeev Khudanpur., 2015. LibriSpeech: an ASR corpus based on public domain audio books. In International Conference on Acoustics, Speech, and Signal Processing, South Brisbane, Queensland, Australia, pp. 5206-5210. 